\title{
Smartphone Application-Based Addiction Among Iranian Adolescents: A Psychometric Study
}

\author{
Chung-Ying Lin ${ }^{1} \cdot$ Vida Imani $^{2} \cdot$ Anders Broström $^{3} \cdot$ Per Nilsen $^{4} \cdot$ Xavier C. C. Fung ${ }^{1}$ • \\ Mark D. Griffiths ${ }^{5}$ - Amir H. Pakpour ${ }^{3,6}$ (D)
}

Published online: 14 November 2018

(C) Springer Science+Business Media, LLC, part of Springer Nature 2018

\begin{abstract}
The Smartphone Application-Based Addiction Scale (SABAS) can be used in screening for the risk of smartphone addiction. This study aimed to validate a Persian version of the SABAS using confirmatory factor analysis (CFA), Rasch analysis, and latent class analysis (LCA). In a sample of 3807 Iranian adolescents, CFAs were used to confirm the factor structure of SABAS, Rasch models were used to examine the unidimensionality of SABAS, and LCAs were used to classify the adolescents in terms of application preferences and smartphone application-based addiction. The unidimensional structure of SABAS was supported by CFA and Rasch model. LCA classified the sample into three subgroups (i.e., low, medium, high) in terms of risk of smartphone addiction. This study showed the unidimensionality of the Persian SABAS with robust psychometric properties. It can be used by healthcare providers in screening for risk of addiction to smartphone applications and provide early intervention if necessary.
\end{abstract}

Keywords Addiction · Adolescent - Internet addiction · Reliability Smartphone addiction · Validity

Amir H. Pakpour

Pakpour_Amir@yahoo.com; apakpour@qums.ac.ir

Chung-Ying Lin

cylin36933@gmail.com

Vida Imani

dr.v.imani@gmail.com

Anders Broström

anders.brostrom@ju.se

Per Nilsen

per.nilsen@liu.se

Xavier C. C. Fung

xavierfungzzz@gmail.com

Mark D. Griffiths

mark.griffiths@ntu.ac.uk 
With ever-improving technology worldwide, almost everyone in developed countries now have smartphones to hand. Although the wide variety of smartphone applications provide convenience and entertainment, research has indicated the potential impact of smartphone use on mental health, including anxiety, depression, and daily dysfunction for a small minority of smartphone users (Demirci et al. 2015; Jeong et al. 2016; Salehan and Negahban 2013). Moreover, studies have revealed that heavy smartphone users are at risk of poor health, both mentally and physically (Kim and Kim 2015; Lee et al. 2014; Munezawa et al. 2011; Yang et al. 2018). For example, overuse of the smartphone and its applications may compromise sleep, and has been associated with various physical symptoms, including musculoskeletal pain, tiredness, headache, and rapid exhaustion (Schoeni et al. 2015; Yang et al. 2017). Lemola et al. (2014) found that adolescents who had their own smartphone tended to have late bedtimes and use their smartphone excessively. The excessive use, in turn, may result in sleep disturbances, poor sleep quality, insomnia symptoms, and depressive symptoms (Lemola et al. 2014; Munezawa et al. 2011).

Although only a small minority of smartphone users display addictive-like symptoms (e.g., Billieux et al. 2015; Elhai et al. 2017a, b; Lopez-Fernandez 2017), healthcare providers cannot ignore the psychological distress associated with problematic smartphone use as illustrated by a recent systematic review of 117 studies (Elhai et al. 2017a, b). However, problematic smartphone use is described and defined inconsistently in the literature, as evidenced by the various terms used including addictive, excessive, compulsive, compensatory, and problematic (Kardefelt-Winther 2014a, b; Widyanto and Griffiths 2006). While there are inconsistent terminologies, problematic smartphone use can be defined as a complex and conceptually composite behavior that causes functional impairment, lack of control, and/or dysfunctional coping (Long et al. 2016).

The issue of smartphone use is complex because smartphones can link to internet and execute various types of applications, which themselves can be addictive (e.g., gaming, gambling, social media use, etc.), and consequently causing psychological impairment. Strong et al. (2018) used a nationally representative sample $(n=3795)$ across five years to examine the associations between internet use, social integration, and depressive symptoms. They found that lowered leisure-time internet use was associated with better social integration and that the growth in internet use was associated with the increased occurrence of depressive symptoms. Moreover, one type of internet activity (i.e., internet gaming) has been proposed as a tentative disorder in the latest version of the Diagnostic and Statistical Manual of Mental Disorders, fifth edition (DSM-5), because of the potential negative effects of addiction to online gaming on wellbeing (American Psychiatric Association 2013).

Given the importance of assessing smartphone addiction, the present study aimed to validate a Persian version of the Smartphone Application-Based Addiction Scale (SABAS) developed by Csibi et al. (2016). The primary benefit of the SABAS is its brevity (six items only) and easy-to-use in screening for the risk of smartphone addiction. Although previous studies found that SABAS is unidimensional, its unidimensionality has never been confirmed using confirmatory factor analysis (CFA) or Rasch analysis. To strengthen the psychometric properties of the SABAS, the present study adopted advanced psychometric testing, involving classical test and modern test theories to verify the unidimensionality of the SABAS. Several related measures on psychological health and addiction were also assessed to provide concurrent validity of the Persian SABAS. In addition, the preferences for smartphone-based applications and the Persian SABAS were used to classify the sample of the present study into different levels of smartphone application preferences. 


\section{Methods}

\section{Participants, Procedure, and Scale Translation Process}

The study procedures were carried out in accordance with the Declaration of Helsinki, and the study was approved by the Ethics committee of Qazvin University of Medical Sciences. All participants were informed about the study, fully understood the study purpose, and all provided informed consent. It should also be noted that parental consent was sought for those participants younger than 18 years of age.

In this methodological study, the data were collected from July 2017 to January 2018. The adolescents were recruited to participate in the study from high schools in Qazvin (a city near to Tehran; population about 400,000; area about $64 \mathrm{~km}^{2}$ ), Iran. A list of high schools was obtained from the Organization for Education in Qazvin. Because the high schools in Iran are all-boys or all-girls schools, 20 all-boys and 20 all-girls schools were selected using a table of random numbers from 64 high schools. From each of these 40 schools, three classes were randomly selected and all adolescents from each class were invited to participate in this study if they fulfilled the inclusion criteria. Adolescents were included in the study if they were aged 1318 years, agreed to participate, and had their own smartphone. Of 4121 adolescents approached, $314(7.62 \%)$ adolescents did not meet inclusion criteria. Consequently, a total of 3807 adolescents participated in the study.

\section{Translation Procedure}

The procedure for translating SABAS was carried out in several steps, according to international recommendations (Beaton et al. 2000; Khoshnevisan et al. 2012). In the first step, the English version of the SABAS (Csibi et al. 2018) was translated into Persian by two bilingual translators whose mother tongue was Persian. In the next step, the translated versions were compared and synthesized by a committee of translators and the research project manager. Once a unified version was completed, it was translated back into English by two bilingual translators. The translators were not aware of the purpose of the study and were blinded from the original English version of the SABAS. An expert committee (including all translators, the project manager, a psychologist, a psychiatrist, a nurse, a psychometrician, and a pediatrician) compared all forward and backward translated versions to consolidate and develop an interim Persian version of the SABAS. The interim Persian version of the SABAS was then piloted on 48 adolescents ( 28 males and 20 females, mean age $=16.01$ years $)$ from other high schools to ensure that the pre-final version retained equivalence in a real situation. Finally, the Persian version of the SABAS was administrated on the study sample of 3807 adolescents.

\section{Instruments}

\section{Smartphone Application-Based Addiction Scale}

The SABAS was first developed in Hungarian (Csibi et al. 2016), and then translated into English, with validation showing satisfactory psychometric properties. The SABAS has a single construct (i.e., addiction to smartphone use) derived from the principal component analysis, high internal consistency $(\alpha=0.81)$, and promising concurrent validity (Csibi et al. 
2018). The SABAS comprises six items, which are rated using a 6-point Likert scale from "Strongly disagree" (1) to "Strongly agree" (6). A higher score in the SABAS indicates that an individual is more at risk of addiction to a smartphone application.

\section{Internet Gaming Disorder Scale-Short Form}

The nine-item Internet Gaming Disorder Scale-Short Form (IGDS9-SF) was developed based on the criteria of internet gaming disorder (IGD) defined in the latest (fifth) edition of the Diagnostic and Statistical Manual of Mental Disorders (DSM-5) (Pontes and Griffiths 2015). The IGDS9-SF has a single construct verified by CFA, high internal consistency $(\alpha=0.90)$, and promising concurrent validity ( $\mathrm{Wu}$ et al. 2017). The nine items of IGDS9-SF are rated using a 5-point Likert scale from "Never" (1) to "Very often" (5). The higher the score on the IGDS-SF9, the more an individual is at risk of IGD.

\section{Nomophobia Questionnaire}

The Nomophobia Questionnaire (NMP-Q), which assesses the fear of being unable to use mobile phone or internet, was developed using a thorough procedure of qualitative and quantitative approaches (Yildirim and Correia 2015). The NMP-Q has a four-factor construct verified by CFA, high internal consistency ( $\alpha=0.70-0.91$ ), and promising concurrent validity (Lin et al. 2018a). The NMP-Q is comprised of 20 items distributed across the four factors of not being able to communicate, losing connectedness, not being able to access information, and giving up convenience. All the items are rated using a 7-point Likert scale from "Strongly disagree" (1) to "Strongly agree" (7). A higher score in the NMP-Q indicates that an individual suffers more from the fear of being unable to use mobile phone or internet.

\section{Bergen Social Media Addiction Scale}

The Bergen Social Media Addiction Scale (BSMAS) (Andreassen et al. 2016) was developed from the Bergen Facebook Addiction Scale (Andreassen et al. 2012) to assess addiction to different types of social media. The BSMAS has a single construct verified by CFA, high internal consistency $(\alpha=0.86)$, and promising concurrent validity (Lin et al. 2017). The BSMAS consists of six items, which are rated using a 5-point Likert scale from "Very rarely" (1) to "Very often" (5). A higher score in the BSMAS indicates that an individual is more at risk of addiction to social media. More specifically, the cutoff of at-risk problematic use of social media in the BSMAS has been proposed at 19 (out of 30) (Bányai et al. 2017).

\section{Depression Anxiety Stress Scale}

The Depression Anxiety Stress Scale (DASS) was developed to assess three types of emotional distress: depression, anxiety, and stress. Given the three types of emotional distress, the DASS has a three-factor construct verified by CFA (Asghari et al. 2008). In addition, the DASS has high internal consistency $(\alpha=0.86-0.89)$ (Pontes and Griffiths 2016). The DASS contains seven items in each type of emotional distress and is rated using a 4-point Likert scale from "Did not apply to me at all" (0) to "Applied to me very much, or most of the time" (3). A higher score in the DASS indicates that an individual suffers more from the specific type of emotional distress. 


\section{ADHD Rating Scale}

The ADHD (Attention Deficit Hyperactivity Disorder) Rating Scale, a parent-rated questionnaire, was developed to capture the symptoms of inattention and hyperactivity that are described in the DSM-IV (Pappas 2006). Given the two symptoms, the ADHD Rating Scale has a two-factor construct verified by CFA (Ghanizadeh and Jafari 2010; Mercier et al. 2016). The ADHD Rating Scale comprises nine items relating to each symptom (i.e., a total of 18 items in the ADHD Rating Scale), and which are rated using a 4-point Likert scale from "Never or not observed" (0) to "Often or always" (3). A higher score in the ADHD Rating Scale indicates that an individual has more inattention or hyperactivity problems.

\section{Data Analysis}

\section{Psychometric Testing Using Classical Test Theory}

The psychometric evaluations using classical test theory included ceiling and floor effects, corrected item-total correlation, internal consistency using Cronbach's $\alpha$, standard error of measurement, test-retest reliability using Pearson correlation, average variance extracted, composite reliability, concurrent validity, CFA, and multigroup CFA (MGCFA). The concurrent validity was tested using Pearson correlation between the SABAS and the following criteria measures: IGDS9-SF, NMP-Q, BSMAS, DASS, and ADHD Rating Scale. In addition, the following cutoffs were proposed to recommend satisfactory psychometric properties of the SABAS: $<20 \%$ in ceiling and floor effects (Jette et al. 2005), $>0.4$ in corrected item-total correlation (Wang et al. 2007), > 0.7 in internal consistency (Cheng et al. 2016), smaller value in standard error of measurement (Cheng et al. 2016), $>0.7$ in test-retest reliability (Lin et al. $2018 \mathrm{~d}$ ), $>0.5$ in average variance extracted (Huang et al. 2013), and $>0.6$ in composite reliability (Huang et al. 2013).

In terms of CFA, the following fit indices were used to determine whether the structure of the SABAS was supported: comparative fit index (CFI) and Tucker-Lewis index (TLI) > 0.9; root mean square error of approximation (RMSEA), and standardized root mean square residual $($ SRMR) $<0.08$ (Cheng et al. 2016; Lin et al. 2012). As for MGCFA, three nested models (configural model, metric invariance model, and scalar invariance model) were tested using the following fit indices: $\triangle \mathrm{CFI}>-0.01, \triangle \mathrm{SRMR}<0.01$, and $\triangle \mathrm{RMSEA}<0.015$ for measurement invariance across subgroups in MGCFA (Chen 2007). The configural model is the baseline model without any constraints in the factor loadings and item intercepts between subgroups; the metric model is based on the configural model to constraint all the factor loadings being equal across subgroups; the scalar model is based on the metric model to constraint all the item intercepts being equal across subgroups (Bagheri et al. 2014).

\section{Psychometric Testing Using Rasch Analysis}

The item difficulty was computed for each item of the SABAS using Rasch analysis. Also, information-weighted fit statistic (infit), mean square (MnSq), and outlier-sensitive fit statistic (outfit) MnSq were applied to examine the fit of each item in the unidimension of the SABAS. More specifically, infit and outfit MnSq with the range between 0.5 and 1.5 indicate that the item fitted the construct of SABAS (Jafari et al. 2012). Moreover, item and person separation reliability were generated based on the Rasch model to indicate the reliability of the SABAS in 
scale-level. Recommended cutoff for both item and person separation reliability is 0.7 (Chang et al. 2014). Differential item functioning (DIF) was further computed in the Rasch model to test the measurement invariance of the SABAS across subgroups at the item level. More specifically, if the subgroup is gender, DIF can clearly identify which item content for males as compared with females are easier or harder to achieve (Lin et al. 2018b, c). The suggested cutoff of DIF is $<0.5$ in the DIF contrast (i.e., the difference of difficulty between two subgroups) (Lin et al. 2018b, c).

\section{Latent Class Analysis and Comparisons Among Different Subgroups}

Two series of latent class analyses (LCA) were performed to determine underlying latent structures of application preferences and application-based risk addiction. The LCA is a mixture modeling technique that assumes that grouping of adolescents based on application preferences and addiction into several homogeneous clusters. The goodness of fit of the model was assessed using Akaike information criteria (AIC), Bayesian information criteria (BIC), and sample size adjusted Bayesian information criterion (SSABIC). The lowest values of these criteria indicate better model fit. These criteria have been shown to be useful for identifying the correct number of classes (Nylund et al. 2007). Furthermore, the accuracy of determining the correct number of classes was addressed by employing the bootstrap likelihood ratio test (BLRT). A significant BLRT indicates that a model with $k$ classes provides a better model fit compared with a model with one less class. Finally, model classification quality was assessed by using entropy. Entropy values range from 0 to 1 with higher values indicating better classification of individuals. Analysis of variance (ANOVA), $\chi^{2}$ test, and Mann-Whitney test were used to compare differences between classes. LCA was performed using Mplus 7.4 (Muthén and Muthén 1998-2017).

\section{Results}

\section{Participant Characteristics}

The mean age among the 3807 participants was 15.53 years $(\mathrm{SD}=1.2)$, their mean hours of smartphone use per week was $5.3(\mathrm{SD}=3.5)$, and slightly over half of them were males $(n=$ $2022 ; 53.1 \%)$. Only a few participants were current smokers $(n=310 ; 8.1 \%)$. Their scores on the questionnaires were 21.3 [out of 45] for IGD9-SF ( $\mathrm{SD}=6.2$ ), 72.6 [out of 140] for NMP-Q $(\mathrm{SD}=16.2), 16.1$ [out of 30] for BSMAS ( $\mathrm{SD}=4.8), 8.2$ [out of 21] for DASS depression subscale $(\mathrm{SD}=4.9), 8.4$ [out of 21] for DASS anxiety subscale $(\mathrm{SD}=5.1), 8.6$ [out of 21] for DASS stress subscale $(\mathrm{SD}=4.6), 4.8$ [out of 27] for inattention subscale of ADHD Rating Scale ( $\mathrm{SD}=2.2$ ), 5.6 [out of 27] for hyperactivity subscale of ADHD Rating Scale ( $\mathrm{SD}=3.0$ ), and 10.4 [out of 54] for total score of ADHD Rating Scale $(\mathrm{SD}=4.0)$ (Table 1).

\section{Psychometric Properties of the SABAS at Item Level}

All the SABAS items had satisfactory item properties, including adequate factor loadings derived from CFA (0.58-0.86), acceptable corrected item-total correlation (0.49-0.85), and excellent test-retest reliability $(r=0.76-0.90)$, as well as appropriate infit $(0.79-1.21)$ and outfit MnSq (0.80-1.26) derived from Rasch model. However, two items displayed substantial 
Table 1 Participants characteristics $(N=3807)$

Mean $\pm \mathrm{SD}$ or $n(\%)$

Age (year)

$15.3 \pm 1.9$

Gender (male)

$2022(53.1)$

Fathers' educational year

$7.9 \pm 4.0$

Mothers' educational year

$6.2 \pm 3.1$

Currently smoker (yes)

$310(8.1)$

Score in Internet Gaming Disorder Scale-Short Form

$21.3 \pm 6.2$

Score in Nomophobia Questionnaire

$72.58 \pm 16.22$

Score in Bergen Social Media Addiction Scale

$16.12 \pm 4.83$

Score in depression ${ }^{\mathrm{a}}$

$8.2 \pm 4.9$

Score in anxiety ${ }^{\mathrm{a}}$

$8.4 \pm 5.1$

$8.6 \pm 4.6$

Score in stress

$5.3 \pm 3.5$

on smartphone use (hour)

$4.75 \pm 2.18$

Score in inattention ${ }^{\mathrm{b}}$

$5.64 \pm 2.95$

Score in hyperactivitiy ${ }^{b}$

$10.42 \pm 4.01$

${ }^{\text {a }}$ Measured using Depression Anxiety Stress Scales

${ }^{\mathrm{b}}$ Measured using ADHD Rating Scale

DIF: Items 6 and 5. For Item 6 ["If I try to cut the time I use my smartphone, I manage to do so for a while, but then I end up using it as much or more than before”'], the DIF displayed across gender indicated that females were prone to score higher on this item than males. For Item 5 ["If I cannot use or access my smartphone when I feel like, I feel sad, moody, or irritable"], the DIF displayed across application preference class indicated that those with low preference of smartphone applications tended to score this item lower than those with high preference of applications. (Please see "Latent class analysis and comparisons among different subgroups" section below for detailed information of the two classes.) Moreover, the difficulty of the item was between -1.69 and 1.27 logit (Table 2).

\section{Psychometric Properties of the SABAS at Scale Level}

Only negligible ceiling effect $(3.2 \%)$ and floor effect $(0.1 \%)$ were found in the SABAS total score. Reliability coefficients shown in using both classical test theory (Cronbach's $\alpha=0.86$; composite reliability $=0.88$ ) and Rasch analyses (person separation reliability $=0.81$; item separation reliability $=1.00$ ) were satisfactory. All the fit statistics derived from CFA indicated excellent fit for the SABAS (CFI =0.980; TLI = 0.966; RMSEA =0.078; SRMR =0.049), and supported the unidimensional structure of the SABAS. Average variance extracted value was acceptable (0.56), and the standard error of measurement was relatively low (2.35). In addition, the test-retest reliability $(r=0.83)$ indicated the high reproducibility of the SABAS total score (Table 3).

Concurrent validity of the SABAS was supported by the significant correlations between SABAS total score and NMP-Q total score $(r=0.35 ; p<0.01)$, ADHD Rating Scale total score $(r=0.67 ; p<0.01)$, DASS stress subscale score $(r=0.32 ; p<0.01)$, DASS anxiety subscale score $(r=0.49 ; p<0.01)$, DASS depression subscale score $(r=0.16 ; p<0.01)$, BSMAS total score $(r=0.34 ; p<0.01)$, and IGDS9-SF total score $(r=0.32 ; p<0.01)$ 
Table 2 Psychometric properties of the Smartphone Application-Based Addiction Scale (SABAS) in item level

\begin{tabular}{|c|c|c|c|c|c|c|c|c|}
\hline \multirow[t]{2}{*}{ Item no. } & \multicolumn{3}{|c|}{ Analyses from classical test theory } & \multicolumn{5}{|c|}{ Analyses from Rasch } \\
\hline & $\begin{array}{l}\text { Factor } \\
\text { loadinga }\end{array}$ & $\begin{array}{l}\text { Item-total } \\
\text { correlation }\end{array}$ & $\begin{array}{l}\text { Test-retest } \\
\text { reliability }\end{array}$ & $\begin{array}{l}\text { Infit } \\
\mathrm{MnSq}\end{array}$ & $\begin{array}{l}\text { Outfit } \\
\text { MnSq }\end{array}$ & Difficulty & $\begin{array}{l}\text { DIF contrast } \\
\text { across gendered }\end{array}$ & $\begin{array}{l}\text { DIF contrast } \\
\text { across application } \\
\text { preference } \\
\text { classes }^{\mathrm{ce}}\end{array}$ \\
\hline SABAS1 & 0.63 & 0.73 & 0.84 & 1.14 & 1.26 & -0.31 & 0.16 & 0.17 \\
\hline SABAS 2 & 0.70 & 0.77 & 0.79 & 1.04 & 1.00 & 0.35 & 0.01 & 0.05 \\
\hline SABAS 3 & 0.58 & 0.68 & 0.90 & 0.79 & 0.75 & 0.34 & 0.27 & 0.24 \\
\hline SABAS 4 & 0.86 & 0.85 & 0.88 & 0.83 & 0.80 & 0.31 & 0.11 & 0.10 \\
\hline SABAS 5 & 0.84 & 0.62 & 0.76 & 0.91 & 0.84 & 0.56 & 0.10 & 0.61 \\
\hline SABAS 6 & 0.82 & 0.49 & 0.85 & 1.21 & 1.24 & -1.20 & -0.62 & 0.11 \\
\hline
\end{tabular}

${ }^{\text {a }}$ Based on confirmatory factor analysis

${ }^{\mathrm{b}}$ Using Pearson correlation

${ }^{c}$ DIF (differential item functioning) contrast $>0.5$ indicates substantial DIF

${ }^{\mathrm{d}}$ DIF contrast across gender $=$ difficulty for females-difficulty for males

${ }^{\mathrm{e}}$ DIF contrast across classes = difficulty for participants with low preference of applications (apps)-difficulty for participants with high preference of apps

$\mathrm{MnSq}$ mean square error

(Table 4). Multi-group CFA demonstrated that male and female adolescents similarly interpreted the structure of SABAS and the content of each item $(\triangle \mathrm{CFI}=-0.003$ and $0.001 ; \triangle \mathrm{SRMR}=0.003$ and $0.008 ; \triangle \mathrm{RMSEA}=0.011$ and 0.003$)$. The measurement invariance was also supported across the two classes of application preference $(\Delta \mathrm{CFI}=-0.002$ and $-0.002 ; \Delta \mathrm{SRMR}=0.018$ and $0.006 ; \Delta \mathrm{RMSEA}=0.006$ and 0.004 ; please see "Latent class analysis and comparisons among different subgroups" section below for detailed information of the two classes) (Table 5).

Table 3 Psychometric properties of the Smartphone Application-Based Addiction Scale in scale level

\begin{tabular}{lll}
\hline Psychometric testing & Value & Suggested cutoff \\
\hline Ceiling effects (\%) & 3.2 & $<20$ \\
Floor effects (\%) & 0.1 & $<20$ \\
Internal consistency (Cronbach's $\alpha$ ) & 0.86 & $>0.7$ \\
Confirmatory factor analysis & & Nonsignificant \\
$\chi^{2}(d f)$ & $217.10(12)^{*}$ & $>0.9$ \\
Comparative fit index & 0.980 & $>0.9$ \\
Tucker-Lewis index & 0.966 & $<0.08$ \\
$\quad$ Root-mean square error of approximation & 0.078 & $<0.08$ \\
$\quad$ Standardized root mean square residual & 0.049 & $>0.5$ \\
Average variance extracted & 0.56 & $>0.6$ \\
Composite reliability & 0.88 & The smaller the better \\
Standard error of measurement & 2.35 & $>0.7$ \\
Item separation reliability from Rasch & 1.00 & $>2$ \\
Item separation index from Rasch & 35.19 & $>0.7$ \\
Person separation reliability from Rasch & 0.81 & $>2$ \\
Person separation index from Rasch & 2.05 & $>0.4$ \\
Test-retest reliability by Pearson correlation & 0.83 & \\
\hline
\end{tabular}

$* p<0.001$ 
Table 4 Zero-order correlations among tested variables

\begin{tabular}{llccccccc}
\hline & SABAS & NMP-Q & ADHD-RS & Stress & Anxiety & Depression & BSMAS & IGDS9-SF \\
\hline SABAS & - & 0.35 & 0.67 & 0.32 & 0.49 & 0.16 & 0.34 & 0.32 \\
NMP-Q & - & - & 0.22 & 0.34 & 0.56 & 0.18 & 0.27 & 0.29 \\
ADHDRS & - & - & - & 0.23 & 0.37 & 0.40 & 0.25 & 0.20 \\
Stress & - & - & - & - & 0.32 & 0.47 & 0.33 & 0.26 \\
Anxiety & - & - & - & - & - & 0.56 & 0.28 & 0.34 \\
Depression & - & - & - & - & - & - & 0.29 & 0.34 \\
BSMAS & - & - & - & - & - & - & - & 0.30 \\
IGDS9-SF & - & - & - & - & - & - & - & - \\
\hline
\end{tabular}

All $p$ values $<0.01$

${ }^{a}$ Depression, anxiety, and stress were measured using Depression Anxiety Stress Scale

ADHD-RS, ADHD Rating Scale; BSMAS, Bergen Social Media Addiction Scale; IGDS9-SF, Internet Gaming Disorder Scale-Short Form; $S A B A S$, Smartphone Application-Based Addiction Scale; NMP-Q, Nomophobia Questionnaire

\section{Latent Class Analysis and Comparisons Among Different Subgroups}

A series of LCAs were performed to classify the adolescents in terms of application preferences and smartphone application-based addiction. The nine types of applications were classified into two classes according to the results of the $\mathrm{LCA}[\mathrm{AIC}=71,742.62, \mathrm{BIC}=$ $71,904.98, \mathrm{SSABIC}=71,822.37$, entropy $=0.87$, and significant BLRT $(p<0.01)]$. The first of the two classes consisted of 1741 participants (46\%) and the second class consisted of 2066 participants (54\%). The two-class model was compared across applications categories based on scores on SABAS, ADHD Rating Scale, BSMAS, IGDS-SF, depression, stress, and anxiety. The results showed that the two-class model was able to differentiate scores of SABAS, depression, anxiety, and IGDS-SF across applications categories (Table 6).

The results of the LCA revealed that the adolescents could be grouped into three classes based on SABAS scores in $(\mathrm{AIC}=11,034.99, \mathrm{BIC}=11,127.65, \mathrm{SSABIC}=11,070.47$, entropy $=0.75$, and significant BLRT $[p<0.01])$. Within these three class groups, the first comprised approximately $38 \%$ of the adolescents (low-risk group, $n=1434$ ), the second $45 \%$ of the adolescents (moderate-risk group, $n=1698$ ), and the third $18 \%$ of the adolescents (high-risk group, $n=675$ ). The three groups of the adolescents were compared across several potential risk factors (Table 7). The adolescents in the high-risk group reported significantly higher scores on the depression, anxiety, and stress subscales, as well as scores on the BSMAS, ADHD, IGDS9-SF, NMP-Q, and SABAS. Furthermore, more than half of adolescents in the high-risk group were male and was significantly more than other groups $(p<0.001)$.

\section{Discussion}

The present study sought to validate a Persian version of SABAS. The study demonstrated that the Persian SABAS, like the Hungarian and English versions of SABAS (Csibi et al. 2016, 2018), had a stable unidimensional structure. The English version of SABAS has only been tested for unidimensionality using an exploratory factor analysis, whereas the present study extended the findings of unidimensionality by applying advanced psychometric methods of CFA and Rasch analysis. In addition to the unidimensionality, the psychometric testing in the 
Table 5 Measurement invariance across gender and across application preference classes on Smartphone Application-Based Addiction Scale through confirmatory factor analysis

\begin{tabular}{|c|c|c|c|c|c|c|c|c|}
\hline \multirow[t]{2}{*}{ Model and comparisons } & \multicolumn{8}{|l|}{ Fit statistics } \\
\hline & $\chi^{2}(\mathrm{df})$ & $\Delta \chi^{2}(\Delta \mathrm{df})$ & CFI & $\Delta \mathrm{CFI}$ & SRMR & $\triangle \mathrm{SRMR}$ & RMSEA & $\triangle \mathrm{RMSEA}$ \\
\hline \multicolumn{9}{|l|}{ Gender } \\
\hline M1: Configural & $500.01(18)^{*}$ & & 0.983 & & 0.027 & & 0.058 & \\
\hline $\begin{array}{l}\text { M2: Plus all } \\
\text { loadings } \\
\text { constrained }\end{array}$ & $583.00(24)^{*}$ & & 0.980 & & 0.030 & & 0.069 & \\
\hline $\begin{array}{l}\text { M3: Plus all } \\
\text { intercepts } \\
\text { constrained }\end{array}$ & $679.04(30)^{*}$ & & 0.979 & & 0.038 & & 0.072 & \\
\hline M2-M1 & & $72.76(6) *$ & & -0.003 & & 0.003 & & 0.011 \\
\hline M3-M2 & & $96.04(6) *$ & & -0.001 & & 0.008 & & 0.003 \\
\hline \multicolumn{9}{|c|}{ Application preference classes ${ }^{\mathrm{a}}$} \\
\hline M1: Configural & $246.58(18)^{*}$ & & 0.974 & & 0.030 & & 0.058 & \\
\hline $\begin{array}{l}\text { M2: Plus all } \\
\text { loadings } \\
\text { constrained }\end{array}$ & $399.92(24) *$ & & 0.972 & & 0.048 & & 0.064 & \\
\hline $\begin{array}{l}\text { M3: Plus all } \\
\text { intercepts } \\
\text { constrained }^{b}\end{array}$ & $571.10(30)^{*}$ & & 0.975 & & 0.054 & & 0.068 & \\
\hline M2-M1 & & $153.34(6)^{*}$ & & -0.002 & & 0.018 & & 0.006 \\
\hline M3-M2 & & $171.18(6)^{*}$ & & -0.002 & & 0.006 & & 0.004 \\
\hline
\end{tabular}

$*_{p}<0.05$

${ }^{a}$ Classified using latent class analysis on the following application preferences: traditional mobile communication, internet-based communication, social media, information, entertainment, games, directions and timetable, lifestyle applications, and health-related applications. Two classes were determined $(n=1741$ for class $1 ; n=$ 2066 for class 2)

M1 model 1, a configural model; $M 2$ model 2, a model based on M1 with all factor loadings constrained being equal across groups; $M 2 P$ model 2 with partial invariance, a model based on M2 with some factor loadings relaxed across groups; $M 3$ mode 3, a model based on M2 or M2P with all item intercepts constrained being equal across groups. CFI, comparative fit index; SRMR, standardized root mean square residual; RMSEA, root mean square error of approximation

present study showed that Persian SABAS had robust psychometric properties including satisfactory internal consistency, excellent test-retest reliability, and negligible floor and ceiling effects among a large sample of Iranian adolescents.

Moreover, to the best of the authors' knowledge, the present study is the first to investigate whether different genders or people with different levels of smartphone application preferences interpret SABAS items differently. It was found that females were more prone to score Item 6 higher than males ("If I try to cut the time I use my smartphone, I manage to do so for a while, but then I end up using it as much or more than before"); adolescents with low preference for smartphone applications tended to score Item 5 lower than those with high preference of smartphone applications ("If I cannot use or access my smartphone when I feel like, I feel sad, moody, or irritable"). Nonetheless, the multigroup CFA demonstrated that the factorial structure of the IGDS-SF9 was the same across gender and across the levels of application preferences.

Females may have different thoughts from males in perceiving the length of time. A study by Rammsayer and Lustnauer (1989) found that males had better perceptions than women in 


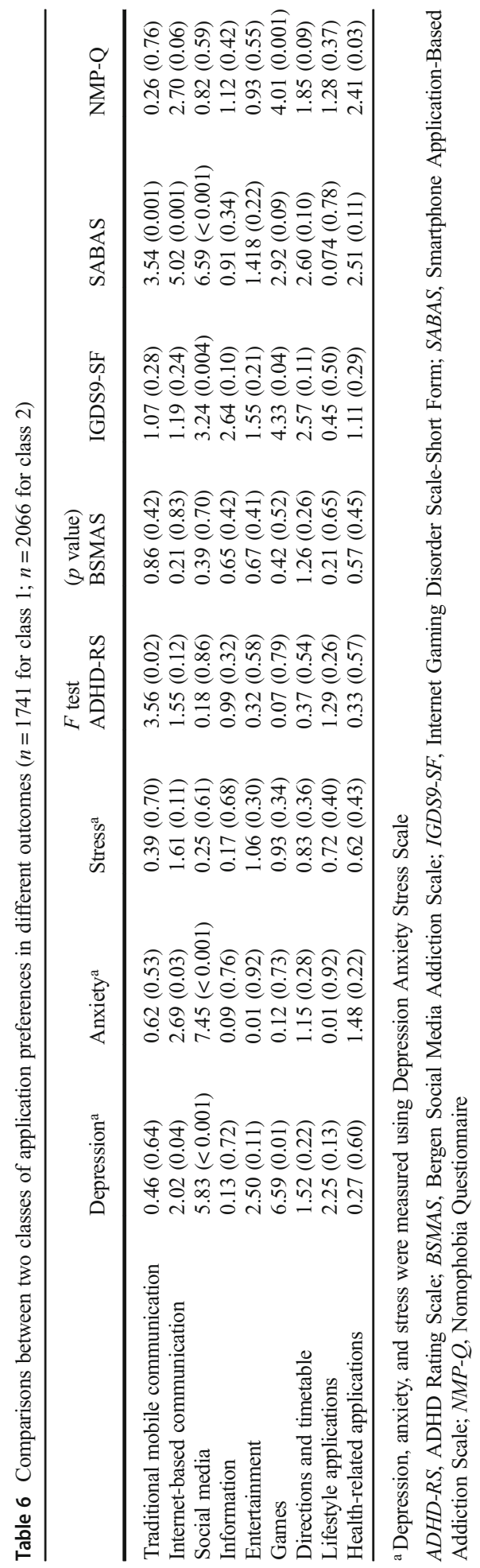


Table 7 Comparisons among three subtypes of participants with different risks of internet addiction

\begin{tabular}{|c|c|c|c|c|c|}
\hline & \multirow{2}{*}{$\begin{array}{l}\text { Low addiction } \\
\text { risk }(n=1434)\end{array}$} & \multirow{2}{*}{$\begin{array}{l}\text { Medium addiction } \\
\text { risk }(n=1698)\end{array}$} & \multirow{2}{*}{$\begin{array}{l}\text { High addiction } \\
\text { risk }(n=675)\end{array}$} & \multicolumn{2}{|c|}{ Overall test } \\
\hline & & & & $F$ test & $p$ value \\
\hline Age in year, mean (SE) & $15.50(0.02)$ & $15.38(0.03)$ & $15.61(0.06)$ & 1.10 & 0.367 \\
\hline Gender $(\text { male } \%)^{*}$ & $23.50(0.02)^{\mathrm{c}}$ & $25.00(0.02)^{\mathrm{c}}$ & $51.50(0.02)^{\mathrm{ab}}$ & 648.40 & $<0.001$ \\
\hline Mother education year & $6.04(0.08)$ & $6.33(0.10)$ & $6.86(0.16)$ & 2.09 & 0.148 \\
\hline Father education year & $7.57(0.09)$ & $7.84(0.10)$ & $8.27(0.15)$ & 0.40 & 0.457 \\
\hline DASS depression score & $7.69(0.13)^{\mathrm{bc}}$ & $8.65(0.12)^{\mathrm{ac}}$ & $10.01(0.24)^{a b}$ & 10.19 & $<0.001$ \\
\hline DASS anxiety score & $7.48(0.04)^{\mathrm{bc}}$ & $8.62(0.03)^{\mathrm{ac}}$ & $9.01(0.05)^{\mathrm{ab}}$ & 15.13 & $<0.001$ \\
\hline DASS stress score & $7.40(0.08)^{b c}$ & $9.14(0.08)^{\mathrm{ac}}$ & $10.11(0.16)^{a b}$ & 10.18 & $<0.001$ \\
\hline BSMAS score & $9.43(0.10)^{\mathrm{bc}}$ & $14.01(0.14)^{\mathrm{ac}}$ & $15.79(0.06)^{\mathrm{ab}}$ & 52.99 & $<0.001$ \\
\hline $\begin{array}{l}\text { ADHD-RS total score, } \\
\text { mean (SE) }\end{array}$ & $9.67(0.14)^{\mathrm{bc}}$ & $12.03(0.16)^{\mathrm{ac}}$ & $13.97(0.27)^{\mathrm{ab}}$ & 106.66 & $<0.001$ \\
\hline $\begin{array}{l}\text { IGDS9-SF total score, } \\
\text { mean (SE) }\end{array}$ & $9.42(0.20)^{\mathrm{bc}}$ & $12.69(0.19)^{\mathrm{ac}}$ & $14.53(0.27)^{\mathrm{ab}}$ & 11.18 & 0.001 \\
\hline NMP-Q & $60.15(0.31)^{\mathrm{bc}}$ & $77.89(0.24)^{\mathrm{ac}}$ & $103.93(0.57)^{\mathrm{ab}}$ & 29.18 & $<0.001$ \\
\hline $\begin{array}{l}\text { SABAS total score, } \\
\text { mean (SE) }\end{array}$ & $13.68(0.08)^{\mathrm{bc}}$ & $21.65(0.06)^{\mathrm{ac}}$ & $32.47(0.12)^{\mathrm{ab}}$ & 109.69 & $<0.001$ \\
\hline
\end{tabular}

DASS, Depression Anxiety Stress Scales; BSMAS, Bergen Social Media Addiction Scale; ADHD-RS, ADHD Rating Scale; IGDS9-SF, Internet Gaming Disorder Scale-Short Form; $N M P-Q$, Nomophobia Questionnaire; $S A B A S$, Smartphone Application-Based Addiction Scale

*Gender variable was analyzed using $\chi^{2}$ test

${ }^{\text {a }}$ Mean difference as compared to the low addiction risk class

${ }^{b}$ Mean difference as compared to the medium addiction risk class

${ }^{\mathrm{c}}$ Mean difference as compared to the high addiction risk class

quantifying time duration. Therefore, it is possible that the male adolescents in the present study interpreted "for a while" in Item 6 differently from female adolescents, and thus, resulted in the different DIF score for Item 6. In terms of the different levels of application preferences, one postulation is that adolescents with high level of smartphone application preferences interpreted "cannot use or access my smartphone" differently from those with low level of smartphone application preferences. Given that adolescents with high level of application preferences tended to use smartphones more, they might have considered using little time of their smartphone as not using. In contrast, those with low level of smartphone application preferences might have considered not using anytime as not using their smartphone. Therefore, the Item 6 displayed DIF across participants with high and low levels of smartphone application preferences. Nevertheless, there was no empirical evidence to support the postulation, and future studies are warranted to examine this further.

Similar to the findings of Csibi et al. (2018), the findings of the present study showed that there were two classes according to the smartphone application preferences. Similarly, communication (including traditional and internet-based methods) and social media were the most relevant smartphone applications influencing the SABAS scores. As Griffiths and Szabo (2014) have pointed out, social networking is one of the most popular online activities, and this may somewhat explain the influences of communication and social media on SABAS scores.

The present study also used SABAS score to classify the sample into three subgroups with different risks of smartphone addiction. Additionally, the three subgroups had significant 
differences in gender, emotional distress, and psychiatric symptoms (e.g., attention and hyperactivity problems). Individuals in the group at high risk of smartphone addiction were generally male, with high emotional distress, and had other addictive internet-related behaviors. These findings are consistent with previous research which has established that excessive smartphone use is associated with being male (e.g., Yang et al. 2018), impaired emotional distress (e.g., Csibi et al. 2018; Yang et al. 2018), and addiction to other behaviors (e.g. Csibi et al. 2018).

There are some limitations to the present study. First, the participants' reports of their first use of a smartphone were not recorded, and this could be a potential confounder to their problematic smartphone use. Second, the diagnosis of any psychiatric symptoms was not obtained from each participant. Therefore, the present study can only demonstrate the association between SABAS and other psychiatric symptoms assessed by questionnaires and was unable to calculate sensitivity and specificity of the SABAS in detecting psychiatric-related diagnosis in adolescents. Third, the psychometric properties of the SABAS were only tested among school students. Therefore, the generalization of the results cannot be applied to people in other age groups (e.g., adults). Based on this specific limitation, future studies should explore the psychometric properties of the SABAS among people across the lifespan. Finally, the present study only recruited Iranian adolescents. Consequently, the SABAS scores cannot be compared across Western and Eastern countries. Given that comparisons across countries or cultures are important, future studies are warranted to investigate the measurement invariance and/or DIF across other countries for the SABAS.

\section{Conclusion}

In conclusion, the present study validated the Persian SABAS using rigorous methods, including a standardized process in translation to ensure the linguistic validity and advanced psychometric testing (e.g., Rasch analysis, CFA, and LCA). By validating the Persian SABAS, the results provide healthcare providers in Persian-speaking countries (approximately 120 million people in Iran, Afghanistan, and Tajikistan) a short, easy-to-use, and easy-to-score tool for screening the risk of addiction to smartphone applications. Although two items displayed substantial DIF (Item 5 for gender and Item 6 for smartphone application preference), results of other psychometric testing all indicated that the Persian SABAS is a reliable and valid instrument. Given the promising psychometric properties, it is recommended that healthcare providers use the SABAS to screen those who are at risk of addiction to smartphone applications and provide early intervention if necessary.

Authors' Contribution AHP and VI created and organized the study and collected the data. C-YL wrote the first draft; AHP analyzed and interpreted the data; MDG supervised the entire study. AB, XCCF, and MDG critically reviewed the manuscript and provided constructive comments. All authors had full access to all data in the study and take responsibility for the integrity of the data and the accuracy of the data analysis. All authors contributed to and have approved the final version of the manuscript.

\section{Compliance with Ethical Standards}

Conflict of Interest The authors declare that they have no conflict of interest. 
Publisher's Note Springer Nature remains neutral with regard to jurisdictional claims in published maps and institutional affiliations.

\section{References}

American Psychiatric Association. (2013). Diagnostic and statistical manual of mental disorders (5th ed.). Washington, DC: Author.

Andreassen, C. S., Torsheim, T., Brunborg, G. S., \& Pallesen, S. (2012). Development of a Facebook addiction scale. Psychological Reports, 110(2), 501-517. https://doi.org/10.2466/02.09.18.PR0.110.2.501-517.

Andreassen, C. S., Billieux, J., Griffiths, M. D., Kuss, D. J., Demetrovics, Z., Mazzoni, E., \& Pallesen, S. (2016). The relationship between addictive use of social media and video games and symptoms of psychiatric disorder: a large-scale cross-sectional study. Psychology of Addictive Behaviors, 30, 252-262. https://doi. org/10.1037/adb0000160.

Asghari, A., Saed, F., \& Dibajnia, P. (2008). Psychometric properties of the Depression Anxiety Stress Scales-21 (DASS-21) in a non-clinical Iranian sample. International Journal of Psychology, 2(2), 82-102.

Bagheri, Z., Jafari, P., Tashakor, E., Kouhpayeh, A., \& Riazi, H. (2014). Assessing whether measurement invariance of the KIDSCREEN-27 across child-parent dyad depends on the child gender: a multiple group confirmatory factor analysis. Global Journal of Health Science, 6(5), 142-153. https://doi.org/10.5539/gjhs. v6n5p 142 .

Bányai, F., Zsila, Á., Király, O., Maraz, A., Elekes, Z., Griffiths, M. D., et al. (2017). Problematic social media use: results from a large-scale nationally representative adolescent sample. PLoS One, 12(1), e0169839. https://doi.org/10.1371/journal.pone.0169839.

Beaton, D. E., Bombardier, C., Guillemin, F., \& Ferraz, M. B. (2000). Guidelines for the process of cross-cultural adaptation of self-report measures. Spine, 25(24), 3186-3191.

Billieux, J., Maurage, P., Lopez-Fernandez, O., Kuss, D. J., \& Griffiths, M. D. (2015). Can disordered mobile phone use be considered a behavioral addiction? An update on current evidence and a comprehensive model for future research. Current Addiction Reports, 2, 156-162. https://doi.org/10.1007/s40429-015-0054-y.

Chang, K.-C., Wang, J.-D., Tang, H.-P., Cheng, C.-M., \& Lin, C.-Y. (2014). Psychometric evaluation, using Rasch analysis, of the WHOQOL-BREF in heroin-dependent people undergoing methadone maintenance treatment: further item validation. Health and Quality of Life Outcomes, 12, 148. https://doi.org/10.1186 /s12955-014-0148-6.

Chen, F. F. (2007). Sensitivity of goodness of fit indexes to lack of measurement invariance. Structural Equation Modeling, 14, 464-504. https://doi.org/10.1080/10705510701301834.

Cheng, C.-P., Luh, W.-M., Yang, A.-L., Su, C.-T., \& Lin, C.-Y. (2016). Agreement of children and parents scores on Chinese version of Pediatric Quality of Life Inventory Version 4.0: further psychometric development. Applied Research in Quality of Life, 11, 891-906. https://doi.org/10.1007/s11482-015-9405-z.

Csibi, S., Demetrovics, Z., \& Szabo, A. (2016). Hungarian adaptation and psychometric characteristics of Brief Addiction to Smartphone Scale (BASS) [in Hungarian]. Psychiatria Hungarica, 31(1), 71-77.

Csibi, S., Griffiths, M. D., Cook, B., Demetrovics, Z., \& Szabo, A. (2018). The psychometric properties of the Smartphone Application-Based Addiction Scale (SABAS). International Journal of Mental Health and Addiction, 16(2), 393-403. https://doi.org/10.1007/s11469-017-9787-2.

Demirci, K., Akgonul, M., \& Akpinar, A. (2015). Relationship of smartphone use severity with sleep quality, depression, and anxiety in university students. Journal of Behavioral Addictions, 4(2), 85-92. https://doi. org/10.1556/2006.4.2015.010.

Elhai, J. D., Dvorak, R. D., Levine, J. C., \& Hall, B. J. (2017a). Problematic smartphone use: a conceptual overview and systematic review of relations with anxiety and depression psychopathology. Journal of Affective Disorders, 207, 251-259. https://doi.org/10.1016/j.jad.2016.08.030.

Elhai, J. D., Levine, J. C., Dvorak, R. D., \& Hall, B. J. (2017b). Non-social features of smartphone use are most related to depression, anxiety and problematic smartphone use. Computers in Human Behavior, 69, 75-82. https://doi.org/10.1016/j.chb.2016.12.023.

Ghanizadeh, A., \& Jafari, P. (2010). Cultural structures of the Persian parents' rating of ADHD. Journal of Attention Disorders, 13, 369-373. https://doi.org/10.1177/1087054709332421.

Griffiths, M. D., \& Szabo, A. (2014). Is excessive online usage a function of medium or activity? An empirical pilot study. Journal of Behavioral Addictions, 3(1), 74-77. https://doi.org/10.1556/JBA.2.2013.016.

Huang, C.-C., Wang, Y.-M., Wu, T.-W., \& Wang, P.-A. (2013). An empirical analysis of the antecedents and performance consequences of using the moodle platform. International Journal of Information and Education Technology, 3, 217-221. https://doi.org/10.7763/IJIET.2013.V3.267. 
Jafari, P., Bagheri, Z., \& Safe, M. (2012). Item and response-category functioning of the Persian version of the KIDSCREEN-27: Rasch partial credit model. Health and Quality of Life Outcomes, 10, 127. https://oi. org/10.1186/1477-7525-10-127.

Jeong, S., Kim, H., Yum, J., \& Hwang, Y. (2016). What type of content are smartphone users addicted to? SNS vs. games. Computers in Human Behavior, 54, 10-17. https://doi.org/10.1016/j.chb.2015.07.035.

Jette, D. U., Warren, R. L., \& Wirtalla, C. (2005). Functional independence domains in patients receiving rehabilitation in skilled nursing facilities: evaluation of psychometric properties. Archives of Physical Medicine and Rehabilitation, 86, 1089-1094. https://doi.org/10.1016/j.apmr.2004.11.018.

Kardefelt-Winther, D. (2014a). A conceptual and methodological critique of internet addiction research: towards a model of compensatory internet use. Computers in Human Behavior, 31, 351-354. https://doi.org/10.1016 j.chb.2013.10.059.

Kardefelt-Winther, D. (2014b). Problematizing excessive online gaming and its psychological predictors. Computers in Human Behavior, 31, 118-122. https://doi.org/10.1016/j.chb.2013.10.017.

Khoshnevisan, A., Yekaninejad, M. S., Ardakani, S. K., Pakpour, A. H., Mardani, A., \& Aaronson, N. K. (2012). Translation and validation of the EORTC brain cancer module (EORTC QLQ-BN20) for use in Iran. Health and Quality of Life Outcomes, 10(1), 54. https://doi.org/10.1186/1477-7525-10-54.

Kim, H.-J., \& Kim, J.-S. (2015). The relationship between smartphone use and subjective musculoskeletal symptoms and university students. Journal of Physical Therapy Science, 27(3), 575-579. https://oi. org/10.1589/jpts.27.575.

Lee, Y.-K., Chang, C.-T., Lin, Y., \& Cheng, Z.-H. (2014). The dark side of smartphone usage: psychological traits, compulsive behavior and technostress. Computers in Human Behavior, 31, 373-383. https://oi. org/10.1016/j.chb.2013.10.047.

Lemola, S., Perkinson-Gloor, N., Brand, S., Dewald-Kaufmann, J. F., \& Grob, A. (2014). Adolescents' electronic media use at night, sleep disturbance, and depressive symptoms in the smartphone age. Journal of Youth and Adolescence, 44(2), 405-418. https://doi.org/10.1007/s10964-014-0176-x.

Lin, C.-Y., Luh, W.-M., Yang, A.-L., Su, C.-T., Wang, J.-D., \& Ma, H.-I. (2012). Psychometric properties and gender invariance of the Chinese version of the self-report Pediatric Quality of Life Inventory version 4.0: short form is acceptable. Quality of Life Research, 21, 177-182. https://doi.org/10.1007/s11136-011-9928-1.

Lin, C. Y., Broström, A., Nilsen, P., Griffiths, M. D., \& Pakpour, A. H. (2017). Psychometric validation of the Persian Bergen Social Media Addiction Scale using classic test theory and Rasch models. Journal of Behavioral Addiction, 6, 620-629. https://doi.org/10.1556/2006.6.2017.071.

Lin, C.-Y., Griffiths, M. D., \& Pakpour, A. H. (2018a). Psychometric evaluation of Persian Nomophobia Questionnaire: differential item functioning and measurement invariance across gender. Journal of Behavioral Addictions, 7, 100-108. https://doi.org/10.1556/2006.7.2018.11.

Lin, C.-Y., Ou, H.-t., Nikoobakht, M., Broström, A., Årestedt, K., \& Pakpour, A. H. (2018b). Validation of Medication Adherence Report Scale (MARS-5) in older stroke patients in Iran. Journal of Cardiovascular Nursing. Advance online publication. https://doi.org/10.1097/JCN.0000000000000488.

Lin, C.-Y., Pakpour, A. H., Broström, A., Fridlund, B., Årestedt, K., Strömberg, A., et al. (2018c). Psychometric properties of the 9-item European Heart Failure Self-Care Behavior Scale using confirmatory factor analysis and Rasch analysis among Iranian patients. Journal of Cardiovascular Nursing, 33(3), 281-288. https://doi. org/10.1097/JCN.0000000000000444.

Lin, Y.-C., Strong, C., Tsai, M.-C., Lin, C.-Y., \& Fung, X. C. C. (2018d). Validating sizing them up: a parentproxy weight-related quality-of-life measure, with community-based children. International Journal of Clinical and Health Psychology, 18, 81-89. https://doi.org/10.1016/j.ijchp.2017.10.001.

Long, J., Liu, T. Q., Liao, Y. H., Qi, C., He, H. Y., Chen, S. B., \& Billieux, J. (2016). Prevalence and correlates of problematic smartphone use in a large random sample of Chinese undergraduates. BMC Psychiatry, 16, 408. https://doi.org/10.1186/s12888-016-1083-3.

Lopez-Fernandez, O. (2017). Short version of the smartphone addiction scale adapted to Spanish and French: towards a cross-cultural research in problematic mobile phone use. Addictive Behaviors, 64, 275-280. https://doi.org/10.1016/j.addbeh.2015.11.013.

Mercier, C., Roche, S., Gaillard, S., Kassai, B., Arzimanoglou, A., Herbillon, V., Roy, P., \& Rheims, S. (2016). Partial validation of a French version of the ADHD-rating scale IV on a French population of children with ADHD and epilepsy. Factorial structure, reliability, and responsiveness. Epilepsy \& Behavior, 58, 1-6. https://doi.org/10.1016/j.yebeh.2016.02.016.

Munezawa, T., Kaneita, Y., Osaki, Y., Kanda, H., Minowa, M., Suzuki, K., Higuchi, S., Mori, J., Yamamoto, R., \& Ohida, T. (2011). The association between use of mobile phones after lights out and sleep disturbances among Japanese adolescents: a nationwide cross-sectional survey. Sleep, 34(8), 1013-1020. https://oi. org/10.5665/SLEEP.1152.

Muthén, L. K., \& Muthén, B. O. (1998-2017). Mplus version 8: user's guide (8th ed.). Los Angeles, CA: Muthén \& Muthén. 
Nylund, K. L., Asparouhov, T., \& Muthén, B. O. (2007). Deciding on the number of classes in latent class analysis and growth mixture modeling: a Monte Carlo simulation study. Structural Equation Modeling, 14, 535-569. https://doi.org/10.1080/10705510701575396.

Pappas, D. (2006). ADHD Rating Scale-IV: checklists, norms, and clinical interpretation. Journal of Psychoeducational Assessment, 24, 172-178. https://doi.org/10.1177/0734282905285792.

Pontes, H. M., \& Griffiths, M. D. (2015). Measuring DSM-5 internet gaming disorder: development and validation of a short psychometric scale. Computers in Human Behavior, 45, 137-143. https://doi. org/10.1016/j.chb.2014.12.006.

Pontes, H. M., \& Griffiths, M. D. (2016). Portuguese validation of the internet gaming disorder scale-short-form. Cyberpsychology, Behavior, and Social Networking, 19, 288-293. https://doi.org/10.1089/cyber.2015.0605.

Rammsayer, T., \& Lustnauer, S. (1989). Sex differences in time perception. Perceptual and Motor Skills, 68(1), 195-198.

Salehan, M., \& Negahban, A. (2013). Social networking on smartphones: when mobile phones become addictive. Computers in Human Behavior, 29, 2632-2639. https://doi.org/10.1016/j.chb.2013.07.003.

Schoeni, A., Roser, K., \& Röösli, M. (2015). Symptoms and cognitive functions in adolescents in relation to mobile phone use during night. PLoS One, 10(7), e0133528. https://doi.org/10.1371/journal.pone.0133528.

Strong, C., Lee, C.-T., Chao, L.-H., Lin, C.-Y., \& Tsai, M.-C. (2018). Adolescent internet use, social integration, and depressive symptoms: analysis from a longitudinal cohort survey. Journal of Developmental and Behavioral Pediatrics, 39(4), 318-324. https://doi.org/10.1097/DBP.0000000000000553.

Wang, Y.-S., Wang, H.-Y., \& Shee, D. Y. (2007). Measuring e-learning systems success in an organizational context: scale development and validation. Computers in Human Behavior, 23, 1792-1808. https://oi. org/10.1016/j.chb.2005.10.006.

Widyanto, L., \& Griffiths, M. D. (2006). 'Internet addiction': a critical review. International Journal of Mental Health and Addiction, 4, 31-51. https://doi.org/10.1007/s11469-006-9009-9.

Wu, T.-Y., Lin, C.-Y., Årestedt, K., Griffiths, M. D., Broström, A., \& Pakpour, A. H. (2017). Psychometric validation of the Persian nine-item Internet Gaming Disorder Scale-Short Form: Does gender and hours spent online gaming affect the interpretations of item descriptions? Journal of Behavioral Addictions, 6 , 256-263. https://doi.org/10.1556/2006.6.2017.025.

Yang, S.-Y., Chen, M.-D., Huang, Y.-C., Lin, C.-Y., \& Chang, C.-H. (2017). Association between smartphone use and musculoskeletal discomfort in adolescent students. Journal of Community Health, 42, 423-430. https://doi.org/10.1007/s10900-016-0271-x.

Yang, S.-Y., Lin, C.-Y., Huang, Y.-C., \& Chang, J.-H. (2018). Gender differences in the association of smartphone use with the vitality and mental health of adolescent students. Journal of American College Health. Advance online publication. https://doi.org/10.1080/07448481.2018.1454930.

Yildirim, C., \& Correia, A.-P. (2015). Exploring the dimensions of nomophobia: development and validation of a self-reported questionnaire. Computers in Human Behavior, 49, 130-137. https://doi.org/10.1016/j. chb.2015.02.059.

\section{Affiliations}

\section{Chung-Ying Lin ${ }^{1} \cdot$ Vida Imani ${ }^{2} \cdot$ Anders Broström $^{3} \cdot$ Per Nilsen ${ }^{4} \cdot$ Xavier C. C. Fung ${ }^{1}$ • Mark D. Griffiths ${ }^{5} \cdot$ Amir H. Pakpour ${ }^{3,6}$}

1 Department of Rehabilitation Sciences, The Hong Kong Polytechnic University, Hung Hom, Hong Kong

2 Pediatric Health Research Center, Tabriz University of Medical Sciences, Tabriz, Iran

3 Department of Nursing, School of Health and Welfare, Jönköping University, Jönköping, Sweden

4 Department of Medical and Health Sciences, Linköping University, Linköping, Sweden

5 International Gaming Research Unit, Psychology Department, Nottingham Trent University, Nottingham, UK

6 Social Determinants of Health Research Center (SDH), Qazvin University of Medical Sciences, Shahid Bahonar Blvd, Qazvin 3419759811, Iran 